\title{
Newborn humans manifest autoantibodies to defined self molecules detected by antigen microarray informatics
}

\author{
Yifat Merbl, ${ }^{1}$ Merav Zucker-Toledano, ${ }^{2,3}$ Francisco J. Quintana, ${ }^{1}$ and Irun R. Cohen' \\ 1Department of Immunology, Weizmann Institute of Science, Rehovot, Israel. 2Pediatric Department, Dana Children's Hospital, \\ Tel-Aviv Sourasky Medical Center, Tel-Aviv, Israel. ${ }^{3}$ Sackler Faculty of Medicine, Tel-Aviv University, Tel-Aviv, Israel.
}

\begin{abstract}
Autoimmune diseases are often marked by autoantibodies binding to self antigens. However, many healthy persons also manifest autoantibodies that bind to self antigens, known as natural autoantibodies. In order to characterize natural autoantibodies present at birth, we used an antigen microarray (antigen chip) to analyze informatically (with clustering algorithms and correlation mapping) the natural IgM, IgA, and IgG autoantibody repertoires present in 10 pairs of sera from healthy mothers and the cords of their newborn babies. These autoantibodies were found to bind to 305 different, mostly self, molecules. We report that in utero, humans develop IgM and IgA autoantibodies to relatively uniform sets of self molecules. The global patterns of maternal IgM autoantibodies significantly diverged from those at birth, although certain reactivities remained common to both maternal and cord samples. Because maternal IgG antibodies (unlike IgM and IgA) cross the placenta, maternal and cord IgG autoantibodies showed essentially identical reactivities. We found that some self antigens that bind cord autoantibodies were among the target self antigens associated with autoimmune diseases later in life. Thus, the obviously benign autoimmunity prevalent at birth may provide the basis for the emergence of some autoimmune diseases relatively prevalent later in life.
\end{abstract}

\section{Introduction}

Natural antibodies are antibodies detected in the absence of known immunization $(1,2)$. Although autoimmunity is forbidden by the clonal selection theory (3), many natural antibodies are autoantibodies; they bind to self molecules. The functions of natural autoantibodies are not clear, but the specific self molecules recognized by these autoantibodies appear to form clinically defining signatures: some autoantibodies create a pattern that heralds susceptibility to a future autoimmune disease, while a different autoantibody pattern can mark resistance to the disease (4). Indeed, it has been proposed that natural autoantibodies and autoreactive $T$ cells in healthy individuals may be directed to a specific and limited set of self molecules; this selective autoimmunity has been termed the immunological homunculus (5-7) or the immunculus (8) - the immune system's internal representation of the body.

In order to characterize natural autoantibodies present at birth, their isotypes, and the self molecules they bind, we used an antigen microarray device to analyze informatically, with clustering algorithms and correlation mapping, the natural $\operatorname{IgM}$, IgA, and IgG autoantibody repertoires present in 10 pairs of sera from healthy mothers and their newborn babies. These autoantibodies were found to bind to 305 different molecules, most of them self molecules. Because only maternal IgG antibodies, but not IgM or IgA antibodies, cross the placenta to the fetus (9-11), the IgM and IgA autoantibodies present in cord serum at birth would have had to arise as a consequence of prenatal immune activation in the baby.

Nonstandard abbreviations used: AIRE, autoimmune regulator; GAD, glutamic acid decarboxylase; MOG, myelin oligodendrocyte glycoprotein; MRI, mean reactivity index.

Conflict of interest: The authors have declared that no conflict of interest exists. Citation for this article: J. Clin. Invest. 117:712-718 (2007). doi:10.1172/JCI29943.
Thus, cord IgM and IgA antibodies originate within the developing baby; cord IgG comes from the mother. We now report that different babies manifested cord IgM autoantibodies binding to a highly correlated, relatively uniform set of self molecules and that cord and maternal IgM reactivities clustered separately. Thus, natural autoimmunity begins in utero in healthy humans, and the prenatal autoantibody repertoires are directed to a specific, standardized set of body molecules, the immunological homunculus (5). Many cord autoantibodies bound self molecules that are associated with major autoimmune diseases later in life. These findings relate to our understanding of both natural autoimmunity and autoimmune disease.

\section{Results}

Analysis by microarray showed that certain autoantibodies were indeed quite prevalent at birth. Table 1 lists the self molecules bound by $\operatorname{IgM}$, IgG, or IgA autoantibodies present in 8 or more of the 10 individual cord sera. Antibody binding to a molecule was scored as positive when the mean intensity of the laser signal was at least 2 standard deviations above the mean background control. Some of the biologic associations of these self antigens are also shown. Table 2 lists the self molecules bound by maternal and cord autoantibodies according to their mean reactivity index (MRI), rather than by their prevalence. The MRI denotes the fold increase (rounded off) above the control of the mean reactivity to the self antigen; the MRI values shown in Table 2 are at least 2 -fold greater than the mean value from PBS incubation alone (see Methods) plus 2 standard deviations in the same sample. For reference, note that the MRI of natural antibodies to E. coli LPS in maternal sera manifested an MRI of 2 for $\operatorname{IgG}$ and $\operatorname{IgA}$ and 5 for IgM (Table 2); thus, some autoantibodies manifested a much higher degree of binding to self molecules than did antibodies to immunogenic foreign LPS. 
Table 1

Common autoantibody repertoire in cord sera

\begin{tabular}{|c|c|c|c|c|}
\hline Self antigens & $\lg M$ & $\lg G$ & $\lg A$ & Associations \\
\hline \multicolumn{5}{|l|}{ Tissues } \\
\hline ssDNA & 10 & - & - & SLE, many others \\
\hline dsDNA & 8 & - & - & SLE, many others \\
\hline GAD & 8 & 10 & - & Type 1 diabetes \\
\hline MOG & 10 & 10 & 10 & Multiple sclerosis \\
\hline Thyroglobulin & 9 & 10 & - & Thyroiditis \\
\hline Myosin & 10 & 10 & - & Myositis \\
\hline Collagen I & 10 & - & - & Anterior uveitis \\
\hline Chorionic gonadotropin & 10 & 10 & - & Infertility \\
\hline Hemoglobin- $\alpha$ & 10 & - & - & Anemia \\
\hline Cytokeratin & 8 & 9 & - & Arthritis \\
\hline \multicolumn{5}{|l|}{ Immune modulators } \\
\hline Galectin 1 & 10 & 10 & - & Antiinflammatory \\
\hline Galectin 3 & 8 & 10 & - & Antiinflammatory \\
\hline Ubiquitin & 9 & - & - & AIRE \\
\hline Gelsolin & 10 & - & 10 & Phagocytosis \\
\hline Annexin & 10 & - & - & Macular function \\
\hline \multicolumn{5}{|l|}{ Hsps } \\
\hline Hsp40 & 10 & 10 & - & Colitis; arthritis \\
\hline Hsp47 & 10 & - & - & Arthritis \\
\hline Hsp60 peptides & 9 & - & - & Diabetes; arthritis \\
\hline$\beta$-Crystallin & 10 & 10 & - & Lens of eye \\
\hline \multicolumn{5}{|l|}{ Plasma proteins } \\
\hline LDL & 10 & 10 & 10 & Atherosclerosis \\
\hline Factor II & 8 & - & - & Blood clotting \\
\hline Factor X & 10 & 10 & 10 & Blood clotting \\
\hline Albumin & 9 & - & - & Carrier; blood volume \\
\hline Fibrin & 10 & - & - & Blood clotting \\
\hline Fibrinogen & 10 & 10 & - & Blood clotting \\
\hline$\beta_{2}$-Microglobulin & 9 & 10 & - & Antigen presenting \\
\hline \multicolumn{5}{|l|}{ Metabolism } \\
\hline Acetylcholinesterase & - & 10 & - & Neurotransmission \\
\hline Catalase & 10 & 10 & - & Antioxidant \\
\hline Protease & 10 & 10 & 8 & Enzyme \\
\hline
\end{tabular}

Shown are self antigens that significantly bound IgM, IgG, and IgA antibodies in 8,9 , or 10 of the 10 individual cord samples. "-" indicates that 7 or fewer samples were reactive to the antigen. dsDNA, doublestranded DNA; ssDNA, single-stranded DNA.

In order to test whether the natural antibodies of different people are indeed directed to a common set of antigens, we used Pearson correlation coefficients to generate a correlation map of the global sets of positive $\operatorname{IgM}, \operatorname{IgA}$, and $\operatorname{IgG}$ reactivities for each of the 10 cord and maternal repertoires compared with the other 9 samples within each group (Figure 1). Correlations, which mathematically depict the degrees of relationship between individual whole repertoires, are represented by intensity of shading from white (correlation coefficient of 1 , perfect correlation) to black (correlation coefficient of 0 , no correlation). The range of lower and upper bounds for a $95 \%$ confidence interval for each group of samples are shown in Table 3. Figure 1 shows that individual maternal $\operatorname{IgM}$, IgA, and IgG repertoires differed from one another; the white diagonal of self identity contrasts with the dark gray background of individual repertoire variability for each antibody isotype. The cords show a different picture: each cord differed from the others in IgG and IgA repertoires, but the cord IgM repertoires were remarkably uniform. The correlations between individual cord $\operatorname{IgM}$ repertoires were so high - so close to white - that the white diagonal of identity marking the perfect correlation of each serum's global reactivity with itself is barely discernible. In other words, the global repertoire of IgM antibodies expressed in any cord is quite similar to that expressed by each of the other 9 cords. We conclude that different babies produce IgM antibodies in utero to a very similar set of self molecules $(12,13)$.

We used clustering algorithms to reveal relationships between defined subsets of the global data; membership in a cluster is determined automatically by an algorithm that mathematically measures shared similarities and dissimilarities. Figure 2 illustrates the results of hierarchical clustering of mother-cord sets (columns) according to each of their IgM and IgA antigen reactivities (rows); the relative degrees of antibody binding are denoted by color, ranging from dark blue to dark red. Note that the range of antibody binding intensity spans several orders of magnitude (see Methods), while the colors are relatively few; thus significantly different reactivities may appear to share the same color. Nevertheless, the representation by color does give some visual sense of the actual clustering. Also note that the positions from top to bottom of particular antigens in the clustering process (Figure 2, rows) were arranged automatically by the clustering algorithm according to relationships between the antigen reactivities; antigens that tend to manifest correlated antibody responses were automatically arranged in adjacent rows. Thus the order of the rows of antigens differs in Figure 2A and Figure 2B because the IgM and IgA repertories show correlated antibody reactivities to different sets of antigens. The clustering of maternal and cord samples shown in Figure 2 demonstrates that the IgM repertoires of the maternal and cord samples differ: the 10 cord IgM repertoires clustered separately from the 10 maternal IgM repertoires (Figure 2A). Thus, the global IgM repertoire of each cord is more similar to the global IgM repertoire of each of the other 9 cords than it is to any of the maternal global repertoires. Note that the maternal samples clustered together separately from the cord samples because the cord samples share a commonality that sets them apart from the maternal samples; the maternal repertoires do differ individually from one another (see Figure 1). In contrast, the IgA repertoires of cord and maternal samples could not be separated into clusters by the unsupervised clustering algorithm applied to the global reactivities to all 305 antigens (Figure 2B). However, we detected differences between the maternal and cord samples as separate groups by selecting a more limited set of 24 of the 305 arrayed antigens, all of which differed significantly between the maternal and cord samples by Wilcoxon rank-sum test (Table 4). Some of the antibodies manifested higher antigen reactivities in the maternal sera and some in the cords; the response to these antigens was not uniformly high or low between the groups. The highest ranking antigens that separated the IgM repertoires (which were significantly different between cord and maternal samples by Wilcoxon rank-sum test) are listed in Table 5. The IgG antibody repertoires of each mother and her newborn separated into essentially identical pairs upon hierarchical clustering (data not shown); this can be explained by the active transport of maternal IgG across the placenta (9-11).

\section{Discussion}

Obviously, the several hundred antigens used in the present study are not an exhaustive list of natural autoantibody self reactivity. Nevertheless, the sample of self molecules, however limited, is illuminating. We concluded that the cord IgM autoantibody repertoire was both selective (only some self molecules are recognized) 
Table 2

MRI of the self molecules bound by maternal and cord autoantibodies

\begin{tabular}{|c|c|c|c|c|c|}
\hline Antibody & Cord IgM & Mother IgM & Cord $\lg A$ & Mother $\lg A$ & Mother/Cord IgG \\
\hline LDL & 57 & 10 & 23 & 11 & 12 \\
\hline Factor $\mathrm{X}$ & 52 & 9 & 17 & 8 & 4 \\
\hline Myosin & 25 & 6 & - & 2 & 11 \\
\hline Gelsolin & 15 & 4 & 6 & 3 & - \\
\hline ssDNA & 10 & 5 & - & - & 3 \\
\hline GAPDH & 6 & - & - & - & 2 \\
\hline Hsp40 & 6 & 6 & - & - & 4 \\
\hline Catalase & 6 & 2 & - & - & 9 \\
\hline Fibrinogen & 5 & 2 & - & - & 9 \\
\hline Ubiquitin & 5 & - & - & - & - \\
\hline Galectin 3 & 4 & 3 & 2 & - & 2 \\
\hline Annexin & 4 & 3 & - & - & - \\
\hline Chorionic gonadotropin & 4 & 5 & - & 2 & 12 \\
\hline Hsp60, peptide 37 & 4 & - & - & - & - \\
\hline Fibrin & 4 & - & - & - & - \\
\hline$\beta$-Cristallin & 4 & 3 & 2 & - & 2 \\
\hline Galectin 1 & 4 & 5 & - & - & - \\
\hline Collagen I & 3 & - & - & - & - \\
\hline Cytokeratin & 3 & - & - & - & 2 \\
\hline GAD & 3 & 3 & 2 & 4 & 12 \\
\hline GST & 3 & 3 & 2 & - & - \\
\hline$\beta_{2}$-Microglobulin & 3 & 2 & - & - & 2 \\
\hline Albumin & 3 & 2 & - & - & - \\
\hline MOG peptide 35-55 & 3 & - & - & - & - \\
\hline Hemoglobin- $\beta$ chain & 3 & 4 & - & - & - \\
\hline Hsp70, peptide 29 & 3 & - & - & - & - \\
\hline Protease & 3 & 3 & - & 5 & 13 \\
\hline Hsp47 & 3 & - & - & - & - \\
\hline PLP & 2 & 2 & - & - & - \\
\hline MOG & 2 & - & 4 & - & 14 \\
\hline Plasmin & 2 & - & - & - & - \\
\hline Factor II & 2 & - & - & - & - \\
\hline ANP & 2 & - & - & - & - \\
\hline Hsp60, peptide 5 & 2 & - & - & - & - \\
\hline AMP & 2 & - & - & - & - \\
\hline Substance P & 2 & - & - & - & - \\
\hline Thyroglobulin & 2 & 7 & - & 4 & 7 \\
\hline Acid phosphatase & 2 & 4 & - & - & 3 \\
\hline $\mathrm{HDL}$ & 2 & - & 19 & 8 & 6 \\
\hline dsDNA & 2 & - & - & - & - \\
\hline Tropomyosin & - & 2 & 2 & - & 2 \\
\hline Thrombin & - & 2 & - & - & - \\
\hline IL-21 & - & 2 & - & - & - \\
\hline Tubulin & - & 2 & - & - & - \\
\hline $\mathrm{C} 1 \mathrm{q}$ & - & - & 2 & - & 4 \\
\hline Acetylcholinesterase & - & 2 & - & - & 2 \\
\hline Collagen X & - & - & - & - & 3 \\
\hline LPSA & - & 5 & - & 2 & 2 \\
\hline Laminin & - & 5 & - & 2 & 3 \\
\hline CA 19-9 & - & 4 & - & - & - \\
\hline Peroxidase & - & 3 & - & 3 & 3 \\
\hline Mucin & - & 3 & - & - & - \\
\hline h MIG & - & 2 & - & - & - \\
\hline Hsp70, peptide 20 & - & 2 & - & - & - \\
\hline Hsp70, peptide 8 & - & 2 & - & - & - \\
\hline Hsp70, peptide 29 & - & 2 & - & - & - \\
\hline Hsp70, peptide 2 & - & 2 & 2 & - & - \\
\hline$\beta_{2}$-Glycoprotein & - & 2 & - & - & - \\
\hline$\alpha_{2}$-Microglobulin & - & - & 15 & 5 & 5 \\
\hline C protein & - & - & - & - & 3 \\
\hline CRF & - & - & - & - & 2 \\
\hline Hsp60 & - & - & - & - & 3 \\
\hline p12 & - & - & - & - & 2 \\
\hline Pepstatin & - & - & 2 & - & - \\
\hline IL-12 & - & - & 2 & - & - \\
\hline Vitronectin & - & - & - & - & 5 \\
\hline
\end{tabular}

Self molecules bound by maternal and cord autoantibodies are listed according to their MRI for cord IgM. MRI values of 2 or greater are shown (an MRI of 1 indicates that mean reactivity was equal to the value of PBS plus 2 standard deviations in the same sample); values less than 2 are indicated as "-." AForeign antigen LPS is included as a reference. AMP, adenosine monophosphate; ANP, atrial natriuretic peptide; CA 19-9, cancer antigen 19-9; GST, glutiathione-S-transferase; h MIG, monokine induced by interferov- $\gamma$; PLP, proteolipid protein. and uniform (different individuals express a common repertoire). Moreover, the common cord IgM repertoire clustered separately from the maternal $\operatorname{IgM}$ repertoires (Figure 2A). Cord IgA was less uniform than was cord IgM, and cord IgA did not cluster separately from maternal IgA; cord and maternal samples, however, differed as groups in their IgA reactivities to a set of 24 antigens (Table 4). The organization of self antigens into correlated sets will be reported in a future study.

Mouthon and associates, using a blot technique to assay natural antibodies binding to undefined antigens in tissue extracts, could not detect the differences in IgM autoantibody repertoires between neonate and adult (demonstrated herein using the antigen chip), nor could their method name the antigens $(12,13)$. Mirilas and coworkers (14) could detect the partial evolution of the natural autoimmune repertoire based on testing a relatively limited number of self antigens.

The present antigen chip findings are compatible with the immunological homunculus hypothesis (5-8) - the idea that the healthy immune system includes autoreactivity to a selected set of particular self molecules. Indeed, the development of our antigen microarray chip (4) and the present work were undertaken to test the homunculus hypothesis. The fact that the primary autoantibody homunculus arises during the uterine life of the fetus, an environment normally free of foreign antigens, suggests that self molecules are likely to serve as the immunogenic stimulus inducing the IgM and IgA homuncular autoantibodies. How this happens is not yet known, but it seems that the development of B cells, like that of $\mathrm{T}$ cells, may involve positive selection for self reactivity (15).

The second disclosure of the present work is that some of the prevalent natural autoantibodies bind self antigens associated with prevalent autoimmune diseases. Tables 1 and 2 reveal that humans are born with autoantibodies to clinically important self antigens, including single-stranded and double-stranded DNA (16), targeted in SLE; glutamic acid decarboxylase (GAD), targeted in type 1 diabetes (17); myelin oligodendrocyte glycoprotein (MOG), targeted in multiple sclerosis (18); cytokeratin, targeted in rheumatoid arthritis (19); and thyroglobulin, targeted in thyroiditis (20). In patients with clinical autoimmune diseases, however, the amounts of the autoantibodies to these self molecules are usually, 


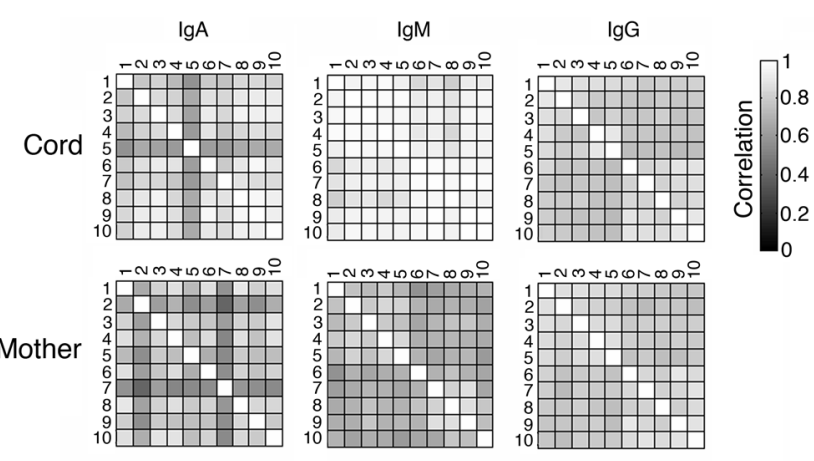

but not always, greater than in healthy individuals; moreover, the autoantibodies associated with autoimmune diseases tend to be predominantly of the $\operatorname{IgG}$ isotype, rather than of the IgM isotype.

Prevalent natural autoantibodies also bind to molecules not known to be associated with autoimmune diseases, such as ubiquitin, galectins, gelsolin, and annexin, which perform critical cellular functions. These molecules participate in cytoskeleton regulation, signal transduction, and proteosome activity, but they also affect immune regulation. Galectins, for example, are immune modulators (21), and gelsolin has been shown to bind the powerful immune activator endotoxin (22); the effects of natural autoantibodies on the functions of these molecules are currently unknown. Hsps also regulate immune reactivity, and $\mathrm{T}$ cell autoreactivity to Hsp molecules appears to downregulate inflammation and autoimmune diabetes and arthritis $(23,24)$. Some antibodies to LDL have been reported to protect against atherosclerosis (25). Thus, autoimmunity to some self molecules might actually serve to prevent autoimmune diseases. Note that much of this self reactivity is of the IgM isotope; it has been proposed that autoimmune diseases might be blocked by natural autoantibodies of the IgM isotype that mask self antigens from pathogenic autoimmune T cells (26).

The self molecule ubiquitin is essential to the destruction and processing of cell molecules; recently it has been reported that a domain of the autoimmune regulator (AIRE) molecule functions as a ligase for ubiquitin. The AIRE gene plays a central role in shaping the $\mathrm{T}$ cell repertoire (27); mutations in AIRE can lead to autoimmune disease (28). Table 2 shows that cord blood, but not maternal blood, contained a relatively high amount of IgM antiubiquitin. It remains to be seen how natural IgM autoantibodies to ubiquitin might influence the immune functions of AIRE in newborns. Other molecules such as plasmin and factor II were targeted by cord IgM, but not by maternal IgM; similarly, the maternal IgM autoimmune repertoire demonstrated reactivities absent from cord serum. Thus different natural autoantibodies appear to mark different stages of human growth and development. If we assume that the mothers began life with IgM autoantibody repertoires similar to those of the cords (Figures 1 and 2), then it would appear that the individuality of one's autoimmune repertoire evolves through one's postnatal immune experience.

The prevalence of cord autoantibodies to enzymes and plasma proteins (Tables 1 and 2 ) also is intriguing; $\beta_{2}$-microglobulin, for example, takes part in antigen presentation to T cells (29). Some natural autoantibodies may have antitumor effects: tyrosinase, involved in the synthesis of melanin, is a melanoma-associated antigen (30), and carbonic anhydrase IX is a tumor-associated antigen in renal cell carcinoma (31).

\section{Figure 1}

Correlation coefficient map comparing each sample with each of the other samples. The 10 cord and 10 maternal samples are shown, and the mean $\lg G$, IgM, and IgA reactivities of the 305 tested antigens were compared to determine correlation. The scale shows the degree of correlation from 0 (black) to 1 (white).

Previously, monoclonal autoantibodies binding to 3 or more different self antigens, termed polyspecific autoantibodies, have been isolated from human fetuses and cord blood (32). These polyspecific autoantibodies were detected in ELISA assays by their binding to actin, tubulin, myosin, thyroglobulin, and/or transferrin and accounted for about $13 \%-16 \%$ of the clones that were isolated. Our microarray study did not test monoclonal antibodies, and so we could not determine the degree of polyspecificity of any of the autoantibodies we detected. However, autoantibodies binding to actin, tubulin or transferrin were not prevalent in our study. Thus the prevalent autoantibodies we detected with our antigen chip technology behaved differently from the classical polyspecific autoantibodies detected by other means.

Because the adaptive immune system has evolved to produce autoantibodies to certain self molecules even before birth, as we have shown here, it is reasonable to conclude that these autoantibodies must provide advantages that offset the occasional autoimmune disease associated with their target autoreactivities (5, 33). It is conceivable that natural autoantibodies, in recognizing specific body molecules, help the immune system gather and integrate essential information about the state of the body's cells (34). In any case, the inclusion of some major disease-associated self antigens in the congenital autoantibody repertoire suggests that pathologic autoimmune disease could arise through a lapse in the regulation of natural, otherwise benign autoimmunity (5).

\section{Methods}

Serum samples. Blood samples were obtained by random availability from 10 healthy women at the onset of labor and from 10 serum samples of the cord blood of their newborns, in the course of normal procedures. All samples were collected with informed consent and approval of the Helsinki committee of the Tel-Aviv Sourasky Medical Center. The newborns were healthy at

\section{Table 3}

Ranges of lower and upper bounds for a 95\% confidence interval for each correlation coefficient

\begin{tabular}{lccc}
\hline & $\lg A$ & $\lg M$ & $\operatorname{Ig} G$ \\
Cord & & & \\
Lower bound & $0.5-0.95$ & $0.76-0.99$ & $0.67-0.88$ \\
Upper bound & $0.65-0.97$ & $0.84-0.99$ & $0.78-0.92$ \\
Mother & & & \\
Lower bound & $0.28-0.88$ & $0.5-0.84$ & $0.67-0.87$ \\
Upper bound & $0.48-0.92$ & $0.64-0.9$ & $0.78-0.92$
\end{tabular}


A

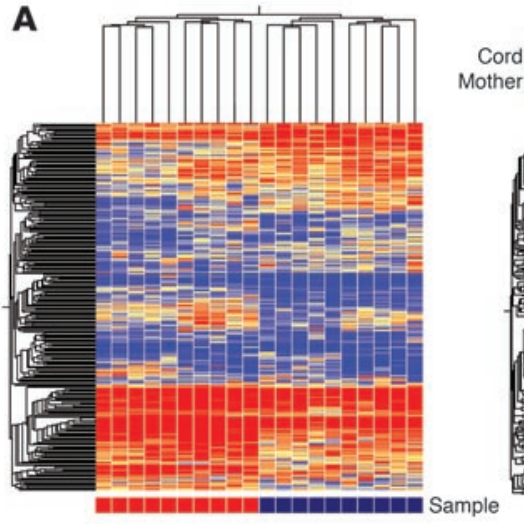

B

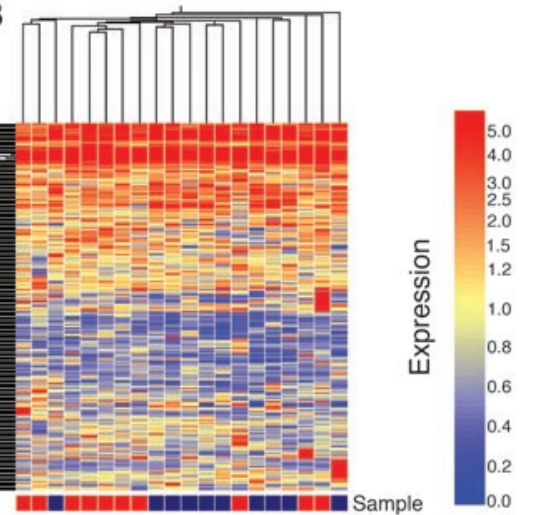

\section{Figure 2}

Clustering of cord and maternal $\lg M$ and $\lg A$. Hierarchical clusterings of $\lg \mathrm{M}(\mathbf{A})$ and $\lg \mathrm{A}(\mathrm{B})$ reactivities for the maternal and cord samples based on their reactivities to each of the 305 antigens in the array. A color code denoting maternal (blue) and cord (red) samples is shown at the bottom. The color scale shows the relative degree of antibody binding from low (dark blue) to high (dark red). the term of pregnancy and appropriate in development and weight for gestational age. The blood samples were allowed to clot at room temperature. After centrifugation, sera were collected and stored at $-20^{\circ} \mathrm{C}$.

Antigens. We spotted 305 antigens on each microarray, as described previously (4). For the most part, we used the same antigens as in our previous studies of natural autoimmune repertoires (4); these included proteins, synthetic peptides from the sequences of key proteins, nucleotides, phospholipids, and other self and non-self molecules. See Supplemental Table 1 (supplemental material available online with this article; doi:10.1172/29943DS1) for the full list.

Antigen microarray. Antigen microarrays were prepared as described previously (4). Briefly, antigens were spotted in replicates of 4 , and the microarrays were blocked for 1 hour at $37^{\circ} \mathrm{C}$ with $1 \%$ bovine serum albumin and incubated under a coverslip overnight at $4^{\circ} \mathrm{C}$ with a $1: 5$ dilution of the test serum in blocking buffer. The quantitative range of signal intensity of binding to each antigen spot was $0.01-65,000$, and this range of detection made it possible to record reliable data with little dilution of test samples. The arrays were then washed and incubated for 1 hour at $37^{\circ} \mathrm{C}$ with a 1:500 dilution of detection antibodies. Three detection antibodies were used: a goat anti-human IgG Cy3-conjugated antibody, a goat antihuman IgM Cy5-conjugated antibody, and a goat anti-human IgA Cy5conjugated antibody (all purchased from Jackson ImmunoResearch Laboratories Inc.). Each sample was analyzed in 2 microarray slides: one with the IgG and IgM fluorescence-labeled anti-isotypes and one with the IgG and IgA fluorescence-labeled anti-isotypes. Thus, the IgG repertoires - which showed no difference between maternal and cord samples because maternal IgG antibodies cross the placenta to the fetus (11) - served as controls for the maternal and cord IgM and IgA determinations measured simultaneously along with the IgG on each plate. Image acquisition by laser and quantification were performed as previously described (4).

Data preprocessing and background filtering. Antigen reactivity was defined by the mean intensity of the 4 replicates binding to that antigen on the microarray. Each chip was normalized by its median reactivity to account for differences in total protein concentrations that affect the background intensity level. We identified positive antibodies (Tables 1 and 2 ) in the following way. In order to establish the minimum level of significant antibody binding, we calculated the mean reactivity level of 32 spots incubated with PBS in place of an antigen on each microarray slide. A signal was scored as positive when it expressed intensity greater than the upper limit of the PBS control, which was defined as the mean intensity of the PBS spots plus twice the standard deviation. Intensity below this background was considered a negative result. The cutoff signal intensity level for each antibody was as follows: cord $\operatorname{IgG}, 4,830$; cord IgM, 1,121; cord IgA, 863; maternal IgG, 4,333; maternal IgM, 5,944; maternal IgA, 2,204. The differ- ence in background intensity between cord and maternal samples in IgM and IgA can be attributed to the relatively greater amounts of total IgM and IgA in maternal blood.

For global pattern analysis, such as in clustering correlation studies, we used the detected intensity of each spot without a threshold intensity cutoff; however, signal intensities that were lower than 0.01 were set to 0.01 and included in the data set.

Data analysis. The processed data set included 3 matrices of IgG, IgA, and $\operatorname{IgM}$ antibody reactivities, each consisting of 305 rows and 30 columns. Each column contained the reactivities measured on a given array (sample), and each row contained the reactivities measured for a given

\section{Table 4}

Antigens to which IgA antibodies separate maternal and cord serum repertoires

\begin{tabular}{lcccc}
\hline Antigen & $\begin{array}{c}\text { Cord } \\
\text { normalized }\end{array}$ & $\begin{array}{c}\text { Mother } \\
\text { normalized }\end{array}$ & H & $\boldsymbol{P}$ \\
Thyroglobulin & 6.497 & 23.26 & $\mathrm{M}$ & 0.0001 \\
Acetylcholinesterase & 1.236 & 5.063 & $\mathrm{M}$ & 0.0001 \\
MOG & 33.31 & 10.1 & $\mathrm{C}$ & 0.0002 \\
Chorionic gonadotropin & 4.187 & 14.37 & $\mathrm{M}$ & 0.0011 \\
HDL & 158.1 & 58.58 & $\mathrm{C}$ & 0.0019 \\
TCR- $\beta$ chain/peptide MED12 & 1.508 & 0.611 & $\mathrm{C}$ & 0.0031 \\
$\alpha_{2}$-Microglobulin & 109.1 & 33.18 & $\mathrm{C}$ & 0.0040 \\
MOG & 1.697 & 4.478 & $\mathrm{M}$ & 0.0040 \\
Fibrinogen & 3.654 & 2.143 & $\mathrm{C}$ & 0.0055 \\
IL-15 & 0.742 & 1.454 & $\mathrm{M}$ & 0.0055 \\
Gelsolin & 51.72 & 23.22 & $\mathrm{C}$ & 0.0070 \\
Elastase & 0.866 & 1.631 & $\mathrm{M}$ & 0.0070 \\
Factor X & 139.3 & 61.94 & $\mathrm{C}$ & 0.0087 \\
GAD & 6.696 & 24.58 & $\mathrm{M}$ & 0.0087 \\
Cholesterol & 0.587 & 2.313 & $\mathrm{M}$ & 0.0087 \\
IL-12 & 0.54 & 0.0433 & $\mathrm{C}$ & 0.0087 \\
Vimentin & 1.428 & 2.745 & $\mathrm{M}$ & 0.0176 \\
Endothelin 1 & 1.9 & 0.719 & $\mathrm{C}$ & 0.0239 \\
Protease & 11.77 & 31.29 & $\mathrm{M}$ & 0.0289 \\
Hsp70, peptide 42 & 0.393 & 1.102 & $\mathrm{M}$ & 0.0289 \\
Gliadin & 2.536 & 5.711 & $\mathrm{M}$ & 0.0343 \\
Galectin 1 & 1.632 & 2.933 & $\mathrm{M}$ & 0.0343 \\
Collagenase & 1.512 & 0.327 & $\mathrm{C}$ & 0.0343 \\
Hemoglobin- $\beta$ chain & 2.249 & 7.72 & $\mathrm{M}$ & 0.0447 \\
& & & & \\
\hline & & & &
\end{tabular}

Shown are the 24 antigens that passed a false discovery rate test with $P<0.05$ (Wilcoxon rank-sum test). Column $\mathrm{H}$ denotes the sample with higher reactivity, mother $(\mathrm{M})$ or cord $(\mathrm{C})$. 
Table 5

Antigens to which $\lg M$ antibodies separate maternal and cord serum repertoires

\begin{tabular}{|c|c|c|c|c|c|c|c|c|c|}
\hline Antigen & $\begin{array}{c}\text { Cord } \\
\text { normalized }\end{array}$ & $\begin{array}{c}\text { Mother } \\
\text { normalized }\end{array}$ & $\mathbf{H}$ & $P$ & Antigen & $\begin{array}{c}\text { Cord } \\
\text { normalized }\end{array}$ & $\begin{array}{c}\text { Mother } \\
\text { normalized }\end{array}$ & H & $P$ \\
\hline C protein & 3.073 & 1.063 & M & 0.0000 & Hsp60, peptide 22 & 0.562 & 0.0733 & C & 0.0015 \\
\hline CA 19-9 & 0.291 & 7.338 & $M$ & 0.0000 & Hsp60, peptide 34 & 1.269 & 0.49 & C & 0.0015 \\
\hline Catalase & 17.66 & 5.998 & C & 0.0000 & MMP1 & 0.891 & 0.342 & C & 0.0015 \\
\hline Factor $\mathrm{X}$ & 167.8 & 24.24 & C & 0.0000 & Thyrocalciterin & 4.8 & 1.092 & C & 0.0015 \\
\hline Fibrin & 10.25 & 2.851 & C & 0.0000 & Myosin & 63.68 & 14.83 & C & 0.0024 \\
\hline Fibrinogen & 17.39 & 4.344 & C & 0.0000 & Substance P & 3.803 & 1.121 & $\mathrm{C}$ & 0.0024 \\
\hline Gelsolin & 42.96 & 8.482 & C & 0.0000 & Glycoprotein 1 & 1.38 & 3.351 & M & 0.0036 \\
\hline MOG & 6.528 & 2.569 & C & 0.0000 & Hsp70, peptide 10 & 0.902 & 1.671 & M & 0.0036 \\
\hline Hsp60, peptide 29 & 1.567 & 0.263 & C & 0.0000 & Actin & 2.608 & 0.962 & C & 0.0049 \\
\hline Hsp70, peptide 14 & 0.954 & 2.465 & M & 0.0000 & Hsp70, peptide 2 & 1.249 & 2.847 & M & 0.0049 \\
\hline LDL & 182.8 & 26.83 & C & 0.0000 & Hsp70, peptide 22 & 0.565 & 1.075 & M & 0.0049 \\
\hline Neuropeptide $Y$ & 3.21 & 0.738 & $\mathrm{C}$ & 0.0000 & Oytocin & 1.657 & 0.813 & $\mathrm{C}$ & 0.0049 \\
\hline Peroxidase & 1.002 & 6.659 & M & 0.0000 & Thrombin & 2.4 & 4.749 & M & 0.0049 \\
\hline IL-6 & 1.433 & 0.587 & C & 0.0000 & $\beta$-Cristallin & 11.15 & 7.052 & $\mathrm{C}$ & 0.0069 \\
\hline Somatostatin & 0.834 & 0.185 & $\mathrm{C}$ & 0.0000 & SOD & 0.948 & 1.764 & $M$ & 0.0069 \\
\hline Spectrin & 0.479 & 0.153 & C & 0.0000 & BMP & 0.636 & 0.327 & C & 0.0098 \\
\hline$\gamma-\mathrm{MSH}$ & 3.045 & 1.305 & C & 0.0001 & IL-15 & 1.129 & 0.526 & C & 0.0098 \\
\hline Hsp60, peptide 12 & 2.032 & 0.56 & $\mathrm{C}$ & 0.0001 & Annexin & 13.62 & 7.332 & $\mathrm{C}$ & 0.0121 \\
\hline Hsp70, peptide 11 & 0.678 & 2.049 & M & 0.0001 & Cardiolipin & 0.248 & 0.096 & $\mathrm{C}$ & 0.0121 \\
\hline MOG peptide 35-55 & 8.487 & 2.625 & C & 0.0001 & Collagenase & 0.387 & 1.978 & M & 0.0121 \\
\hline LPSA & 0.878 & 13.27 & $M$ & 0.0003 & Endothelin 2 & 0.224 & 0.0554 & $\mathrm{C}$ & 0.0121 \\
\hline Thyroglobulin & 5.369 & 15.07 & M & 0.0003 & Factor II & 4.724 & 2.128 & C & 0.0121 \\
\hline Collagen I & 9.69 & 2.191 & C & 0.0004 & Hsp60, peptide 18 & 0.463 & 0.163 & C & 0.0121 \\
\hline Hsp47 & 8.276 & 2.671 & $\mathrm{C}$ & 0.0004 & Hsp70, peptide 37 & 0.653 & 1.02 & M & 0.0121 \\
\hline Hsp60, peptide 37 & 8.676 & 2.31 & C & 0.0004 & Plasmin & 3.982 & 1.385 & $\mathrm{C}$ & 0.0121 \\
\hline Hsp60, peptide 35 & 0.771 & 0.212 & C & 0.0008 & $\mathrm{C} 1 \mathrm{q}$ & 1.748 & 3.265 & M & 0.0151 \\
\hline AMP & 6.848 & 1.154 & C & 0.0011 & Enolase & 0.548 & 1.397 & $M$ & 0.0151 \\
\hline Big gastrin I & 0.891 & 0.266 & C & 0.0011 & Hsp60, peptide 26 & 0.578 & 0.277 & C & 0.0151 \\
\hline Acetylcholinesterase & 2.668 & 5.814 & $M$ & 0.0011 & Hsp70, peptide 7 & 0.637 & 0.242 & $\mathrm{C}$ & 0.0151 \\
\hline Hsp60, peptide 28 & 0.67 & 0.177 & C & 0.0011 & Neurotensin & 1.394 & 0.701 & $\mathrm{C}$ & 0.0151 \\
\hline Hsp70, peptide 30 & 1.194 & 3.103 & M & 0.0011 & PLP & 5.222 & 2.243 & $\mathrm{C}$ & 0.0151 \\
\hline Hsp70, peptide 42 & 0.487 & 2.159 & M & 0.0011 & Cytokeratin & 8.134 & 2.229 & M & 0.0198 \\
\hline Mucin & 0.902 & 3.871 & $M$ & 0.0011 & Hsp60, peptide 17 & 0.794 & 0.194 & $\mathrm{C}$ & 0.0198 \\
\hline MBP & 1.175 & 0.351 & $\mathrm{C}$ & 0.0011 & Elastase & 0.689 & 0.334 & $\mathrm{C}$ & 0.0255 \\
\hline ssDNA & 29.12 & 11.23 & $\mathrm{C}$ & 0.0011 & Hsp70, peptide 33 & 0.457 & 0.803 & $M$ & 0.0255 \\
\hline Ubiquitin & 10.07 & 1.32 & $\mathrm{C}$ & 0.0011 & Hsp70, peptide 41 & 0.371 & 0.751 & M & 0.0255 \\
\hline$\beta_{2}$-Microglobulin & 8.456 & 3.449 & $\mathrm{C}$ & 0.0015 & & & & & \\
\hline
\end{tabular}

Shown are the 73 antigens that passed a false discovery rate test with $P<0.03$. Column $\mathrm{H}$ denotes the sample with higher reactivity, mother (M) or cord (C). AForeign antigen LPS is included as a reference. MBP, myelin basic protein; $\gamma$-MSH, $\gamma$-melanocyte-stimulating hormone; PLP, proteolipid protein; SOD, superoxide dismutase.

antigen over all arrays. Data were normalized by dividing each array by its median reactivity; this made it possible to transform different arrays to comparable reactivity levels.

Clustering. Raw data were normalized and analyzed using GeneSpring software (version 7; Silicon Genetics) and MATLAB (version 6.5.0.180913a, release 13; The MathWorks). Clustering analysis was performed for each isotype separately to test samples from the different groups (cord and mother). The Euclidean distance measure was used to detect related samples and related antigens, which are not discussed here. The results of clustering were robust in a leave-one-out test (35). In addition, we used a Wilcoxon ranksum test (36) to identify antigens that separated each of the groups (see Tables 4 and 5). The Benjamini and Hochberg false discovery rate method (37) was applied, using a $P$ value of 0.05 to determine significance.

Correlation mapping. Using the mean antibody reactivity value of each sample, we calculated the common Pearson correlation coefficient between that sample and each of the other samples within the cord sera and mater- nal sera groups. The correlation coefficients from 0 to 1 were expressed using a black and white color bar with gradations of gray (Figure 1). The range of lower and upper bounds for a $95 \%$ confidence interval for each of the correlation coefficients were evaluated and are shown in Table 3.

\section{Acknowledgments}

We thank Shirley Horn-Saban and Ron Ophir (Weizmann Institute of Science) for technical support. A grant from the Center for the Study of Emerging Diseases, Jerusalem, supported part of this study.

Received for publication August 21, 2006, and accepted in revised form December 26, 2006.

Address correspondence to: Irun R. Cohen, Department of Immunology, Weizmann Institute of Science, Rehovot 76100, 
Israel. Phone: 972-8-934-2911; Fax: 972-8-934-4103; E-mail: irun.cohen@weizmann.ac.il.
Francisco J. Quintana's present address is: Center for Neurologic Diseases, Harvard Medical School, Boston, Massachusetts, USA.
Yifat Merbl's present address is: Systems Biology Department, Harvard Medical School, Boston, Massachusetts, USA.
Yifat Merbl and Merav Zucker-Toledano contributed equally to this work.
1. Cohen, I.R., and Norins, L.C. 1966. Natural human antibodies to gram-negative bacteria: immunoglobulins G, A, and M. Science. 152:1257-1259.

2. Coutinho, A., Kazatchkine, M.D., and Avrameas, S 1995. Natural autoantibodies. Curr. Opin. Immunol. 7:812-818.

3. Burnet, F.M. 1972. Auto-immunity and autoimmune disease. F.A. Davis Company. Philadelphia, Pennsylvania, USA. 243 pp.

4. Quintana, F.J., et al. 2004. Functional immunomics: microarray analysis of IgG autoantibody repertoires predicts the future response of mice to induced diabetes. Proc. Natl. Acad. Sci. U. S. A. 101(Suppl. 2):14615-14621.

5. Cohen, I.R. 1992. The cognitive paradigm and the immunological homunculus. Immunol. Today. 13:490-494

6. Cohen, I.R., and Young, D.B. 1991. Autoimmunity, microbial immunity and the immunological homunculus. Immunol. Today. 12:105-110.

7. Nobrega, A., et al. 1993. Global analysis of antibody repertoires. II. Evidence for specificity, selfselection and the immunological "homunculus" of antibodies in normal serum. Eur. J. Immunol. 23:2851-2859.

8. Poletaev, A.B. 2002. The immunological homunculus (immunculus) in normal state and pathology. Biochemistry Mosc. 67:600-608

9. Mostov, K.E. 1994. Transepithelial transport of immunoglobulins. Annu. Rev. Immunol. 12:63-84.

10. Saji, F., Samejima, Y., Kamiura, S., and Koyama, M. 1999. Dynamics of immunoglobulins at the fetomaternal interface. Rev. Reprod. 4:81-89.

11. Simister, N.E. 2003. Placental transport of immunoglobulin G. Vaccine. 21:3365-3369.

12. Mouthon, L., et al. 1995. Invariance and restriction toward a limited set of self-antigens characterize neonatal IgM antibody repertoires and prevail in autoreactive repertoires of healthy adults. Proc. Natl. Acad. Sci. U. S. A. 92:3839-3843.

13. Mouthon, L., et al. 1996. The self-reactive antibody repertoire of normal human serum IgM is acquired in early childhood and remains conserved through- out life. Scand. J. Immunol. 44:243-251.

14. Mirilas, P., Fesel, C., Guilbert, B., Beratis, N.G., and Avrameas, S. 1999. Natural antibodies in childhood: development, individual stability, and injury effect indicate a contribution to immune memory. J. Clin. Immunol. 19:109-115.

15. Hayakawa, K., et al. 1999. Positive selection of natural autoreactive B cells. Science. 285:113-116

16. Pisetsky, D.S. 2000. Anti-DNA and autoantibodies. Curr. Opin. Rheumatol. 12:364-368.

17. Kaufman, D.L., et al. 1993. Spontaneous loss of T-cell tolerance to glutamic acid decarboxylase in murine insulin-dependent diabetes. Nature. 366:69-72.

18. Iglesias, A., Bauer, J., Litzenburger, T., Schubart, A. and Linington, C. 2001. T- and B-cell responses to myelin oligodendrocyte glycoprotein in experimental autoimmune encephalomyelitis and multiple sclerosis. Glia. 36:220-234.

19. Sharma, B.L., Rani, R., Misra, R., and Aggarwal, A 2000. Anti-keratin antibodies in patients with rheumatoid arthritis. Indian J. Med. Res. 111:215-218.

20. Bouanani, M., et al. 1991. Autoimmunity to human thyroglobulin. Respective epitopic specificity patterns of anti-human thyroglobulin autoantibodies in patients with Sjogren's syndrome and patients with Hashimoto's thyroiditis. Arthritis Rheum. 34:1585-1593.

21. Liu, F.T. 2005. Regulatory roles of galectins in the immune response. Int. Arch. Allergy Immunol. 136:385-400

22. Bucki, R., et al. 2005. Inactivation of endotoxin by human plasma gelsolin. Biochemistry. 44:9590-9597.

23. Quintana, F.J., Carmi, P., Mor, F., and Cohen, I.R. 2004. Inhibition of adjuvant-induced arthritis by DNA vaccination with the $70-\mathrm{kd}$ or the $90-\mathrm{kd}$ human heat-shock protein: immune cross-regulation with the $60-\mathrm{kd}$ heat-shock protein. Arthritis Rheum. 50:3712-3720.

24. Quintana, F.J., Carmi, P., Mor, F., and Cohen, I.R. 2002. Inhibition of adjuvant arthritis by a DNA vaccine encoding human heat shock protein 60 J. Immunol. 169:3422-3428.
25. Su, J., et al. 2005. Antibodies of IgM subclass to phosphorylcholine and oxidized LDL are protective factors for atherosclerosis in patients with hypertension. Atherosclerosis. 188:160-166.

26. Cohen, I.R., and Cooke, A. 1986. Natural autoantibodies might prevent autoimmune disease. Immunol. Today. 7:363-364.

27. Anderson, M.S., et al. 2002. Projection of an immunological self shadow within the thymus by the aire protein. Science. 298:1395-1401.

28. Nagamine, K., et al. 1997. Positional cloning of the APECED gene. Nat. Genet. 17:393-398.

29. York, I.A., and Rock, K.L. 1996. Antigen processing and presentation by the class I major histocompatibility complex. Annu. Rev. Immunol. 14:369-396.

30. Goldberg, S.M., et al. 2005. Comparison of two cancer vaccines targeting tyrosinase: plasmid DNA and recombinant alphavirus replicon particles. Clin. Cancer Res. 11:8114-8121.

31. Staehler, M., Rohrmann, K., Haseke, N., Stief, C.G., and Siebels, M. 2005. Targeted agents for the treatment of advanced renal cell carcinoma. Curr. Drug Targets. 6:835-846.

32. Guigou, V., et al. 1991. Ig repertoire of human polyspecific antibodies and B cell ontogeny. J. Immunol. 146:1368-1374.

33. Avrameas, S. 1991. Natural autoantibodies: from 'horror autotoxicus' to 'gnothi seauton'. Immunol. Today. 12:154-159.

34. Cohen, I.R. 2000. Tending Adam's garden: evolving the cognitive immune self. Academic Press Inc. London, United Kingdom. 296 pp.

35. Ben-Dor, A., et al. 2000. Tissue classification with gene expression profiles. J. Comput. Biol. 7:559-583.

36. Wilcox, R.R. 2001. Fundamentals of modern statistical methods: substantially improving power and accuracy. Springer. New York, New York, USA. 328 pp.

37. Benjamini, Y., and Hochberg, Y. 1995. Controlling the false discovery rate: a practical and powerful approach to multiple testing. Journal of the Royal Statistical Society (Series B). 57:289-300. 